\title{
Engineering "JiaoJiao" (maltose syrup) with chopsticks: From traditional Chinese sweet food to skin-like iontronics
}

\author{
Jiahui Huang ${ }^{1}$ and Peiyi $\mathrm{Wu}^{1,2^{*}}$
}

\begin{abstract}
The advancement of technology has had a profound impact on all areas of life, with an ever more intimate integration of the digital and biological spheres, but it may also be accompanied by an environmental crisis caused by the abuse of large quantities of electronics and petrochemicals. Next-generation "green" electronics or iontronics with high biocompatibility, biodegradation, low cost and mechanical compliance promise to mitigate these adverse effects, but are often limited by the finite choices of materials and strategies. Herein, maltose syrup, a traditional water-dissolvable saccharide food called "JiaoJiao" in Chinese, is engineered to replace unsustainable conductive components of current electronic devices. After churning and pulling with two chopsticks, known as aeration, the aerated maltose syrup has optimized viscoelasticity, mechanical adaptation, robustness, remodeling and self-healing capability, yet with transient behavior. Moreover, the structural and viscoelastic evolution during aeration is also analyzed to maximize the contribution from structures. As a proof-of-concept, a type of "green" skinlike iontronics is prepared, which exhibits reliable strain sensing ability and is subsequently applied for intelligent information encryption and transmission based on a novel concept of sending Morse code. This work greatly extends the current material choice and is expected to shed light on the development of a sustainable future.
\end{abstract}

Keywords: green electronics, skin-like iontronics, sustainable, viscoelastic, transient electronics

\section{INTRODUCTION}

Amid the rapid flourishment of Internet of Things technology, electronic devices have emerged as one of the critical drivers for the global digitalization economy, displaying huge potential and added value to various realms such as artificial intelligence, wearable devices, healthcare and robotics [1-3]. These advances, however, often come at the cost of negative impacts on our ecosystem, from production to improper disposal [4-6]. For example, electronic devices are traditionally designed with mechanical and chemical durability in mind and comprised of potentially toxic compounds. Thus, they cannot be easily disposed of after damage or obsolescence, and the resulting electronic waste poses a huge burden on the environment. In response to these issues, the construction of electronic devices from sustainable or biodegradable materials that can decompose to harmless by-products, known as "green" electronics, is becoming a topic of great interest [5-9].

Recently developed transient electronics that can maintain structural stability and achieve high performance during use and degrade in a relatively short time on demand offer new opportunities [10]. To date, some degradable or dissolvable polymers based on polylactic acid, poly(lactic-co-glycolic acid) and polyvinyl alcohol have been widely introduced in the preparation of transient electronics $[11,12]$. However, the high modulus and poor elasticity of these linear polymers limit their further applications in wearable electronics. Alternatively, bioderived materials such as cellulose derivatives [13,14] and silk [15-17], although cheap and biodegradable, usually fail to maintain mechanical integrity under high loads [18]. Therefore, challenges here originate from the multiple demands on the materials, from mechanical compliance and durability to low cost, high processability, yet

\footnotetext{
${ }^{1}$ State Key Laboratory of Molecular Engineering of Polymers, Department of Macromolecular Science and Laboratory for Advanced Materials, Fudan University, Shanghai 200433, China

${ }^{2}$ State Key Laboratory for Modification of Chemical Fibers and Polymer Materials, College of Chemistry, Chemical Engineering and Biotechnology, Center for Advanced Low-Dimension Materials, Donghua University, Shanghai 201620, China

* Corresponding author (email: peiyiwu@fudan.edu.cn)
} 
fully biodegradable.

Traditional foods, after thousands of years' explorations, already possess mature formulas, affording unexplored possibilities towards "green" electronics or iontronics [19-23]. Meanwhile, some foods also own skin-like mechanical properties, interesting rheological properties, good transparency and edibility. For example, Jiang and co-workers $[19,24]$ developed fully food-based edible nutritive electronics and supercapacitors. Still, when it comes to practicality and long-term usability, one of the biggest problems in food storage is spoilage, which is mainly caused by dehydration and the multiplication of microorganisms [25]. Maltose syrup, on the other hand, an ancient and famous sweet food made from malt and glutinous rice, known as "JiaoJiao" in Chinese, is an appealing candidate to meet these challenges. It is stable over long periods of time due to its low hygroscopicity and high osmotic pressure, while its superb water solubility endows it with transient behavior.

Here, learning from the food aeration technology over 6000 years, we engineer viscoelastic aerated maltose syrup simply by manual aeration with chopsticks. The resulting aerated maltose syrup is mechanically adaptive, selfhealing, and swiftly dissolved in water. Moreover, we also analyze the structural and viscoelastic evolution during aeration to maximize the contribution from structures. Further, demonstration of this material in skin-like iontronics that exhibit reliable capability for transmitting the "sweet" Morse code illustrates its great potential for nextgeneration "green" electronics. We anticipate that edible, biodegradable, and recyclable food-based electronic devices would be a frontier field with infinite possibilities for a sustainable future in the Internet of Things, as well as encourage us to seek new insights from nature to design novel materials.

\section{EXPERIMENTAL SECTION}

\section{Materials}

The maltose syrup was obtained from a handmade shop. $\mathrm{NaCl}$ was purchased from Aladdin Chemical Co.

\section{Preparation of viscoelastic maltose syrup}

An appropriate amount of maltose syrup (ca. $5 \mathrm{~g}$ ) was taken out with two chopsticks. The two chopsticks wrapped with maltose syrup constantly pulled and churned for about $30 \mathrm{~min}$. The viscoelastic aerated maltose syrup was finally got and could be manipulated into various shapes with our hands. To achieve ionic conductivity, ionic salt (ca. $2 \mathrm{wt} \% \mathrm{NaCl}$ ) was added into the viscoelastic maltose syrup by kneading.

\section{Evaluation of the water-retaining performance of maltose syrup}

Maltose syrup was left at room temperature $\left(22^{\circ} \mathrm{C}, 40 \%\right.$ $\mathrm{RH})$ without any external coverage. The original weight of maltose syrup was marked as $W_{0}$, and the instant weight after certain storage durations was marked as $W$. $W / W_{0}(\%)$ was a parameter to assess the water-retaining ability of maltose syrup.

\section{Devices fabrication}

A dielectric layer (VHB, 3M 4905) was sandwiched between two ionic conductors (aerated maltose syrup layers) that were connected to two $\mathrm{Cu}$ wires, resulting in a capacitive sensor. When the device was applied as a finger motion sensor and Morse code transmitter, it was sealed between two layers of VHB tape and then attached to a finger.

\section{Characterization of raw materials}

The following measurements used maltose syrup or aerated maltose syrup directly, and no extra work was required. Rheological measurements were taken on a HAAKE MARS modular advanced rheometer and parallel plates with a diameter of $25 \mathrm{~mm}$ were used. Dynamic frequency sweep measurements were performed at $15^{\circ} \mathrm{C}$ in an oscillation mode using a fixed oscillatory strain of $0.5 \%$ and a fixed gap of $1 \mathrm{~mm}$. Viscometry was conducted at a shear rate of $1 \mathrm{~s}^{-1}$ at $15^{\circ} \mathrm{C}$. The optical micrographs were taken on a polarized optical microscope (Leica DM2500P). The following tests were performed by lyophilizing the sample before testing. The microstructures and morphology of the maltose syrup at different time points were observed by field emission scanning electron microscopy (FESEM, Zeiss Ultra 55 with EDX). Fourier transformed infrared (FTIR) spectra were performed on a Nicolet 6700 spectrometer by using attenuated total reflectance (ATR) method.

\section{Characterization of the aerated maltose syrup-based skin- like iontronics}

The capacitance measurements were collected by an LCR meter (TH2830) with the sweeping frequency of $1 \mathrm{kHz}$ and the alternating-current (AC) voltage of $1 \mathrm{~V}$. The LCR meter was also applied to record the real-time capacitive changes under various stimuli. Capacitance-strain curves of the device were recorded at a deformation rate of $10 \mathrm{~cm} \min ^{-1}$ performed on a universal tensile machine (Instron 5966, USA). 


\section{RESULTS AND DISCUSSION}

\section{Optimization of micro-structure and rheological} properties of "JiaoJiao" (maltose syrup) with chopsticks

Maltose syrup is a kind of edible and popular saccharide food in China, commonly known as "JiaoJiao". It is often produced by traditional fermentation methods, with malt and glutinous rice as raw materials, through a two-step process of liquefaction and saccharification (Figs S1 and S2), resulting in a mixture composed of maltose together with varying amounts of glucose, oligosaccharides, and maltodextrins [26,27]. On the other hand, aeration technology is an important tool in food processing. The wide variety of foods we eat daily contain an indispensable ingredient not listed on the label, and that is air. Pulling, probably the most common method of aeration, is the process of bringing air into the matrix by repeated stretching, folding and layering of the substrate. Introducing a gas phase into a food matrix not only affects its texture to make the product lighter, but also changes its appearance, color and mouth-feel $[28,29]$. As a result, aerated foods, which are more than 6000 years old, now feature within confectionery, dairy products, baked goods, and so on. The technology also provides the foundation for modern foam science and its diverse applications [30].

Learning from the food aeration technology, we used two chopsticks to manually aerate the maltose syrup by churning and pulling to acquire aerated maltose syrup with optimal viscoelasticity (Fig. 1a). We first took ca. $5 \mathrm{~g}$ maltose syrup, churned and pulled incessantly for about $30 \mathrm{~min}$, and then observed changes in appearance every 10 min. As shown in Fig. 1b, during aeration, the maltose syrup climbed along the chopsticks in an effect referred to as Weissenberg effect, which is one of the intriguing features of non-Newtonian fluid [31]. In Fig. 1c and Movie S1, the pristine maltose syrup was translucent without impurities, highly viscous but with certain fluidity, and the color was light yellow or brownishyellow. With the increased aeration time, air was continually incorporated into the mass, and both the color and volume of maltose syrup changed to different de-

a

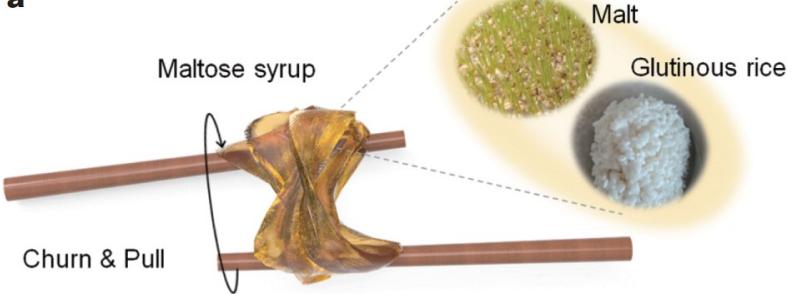

b
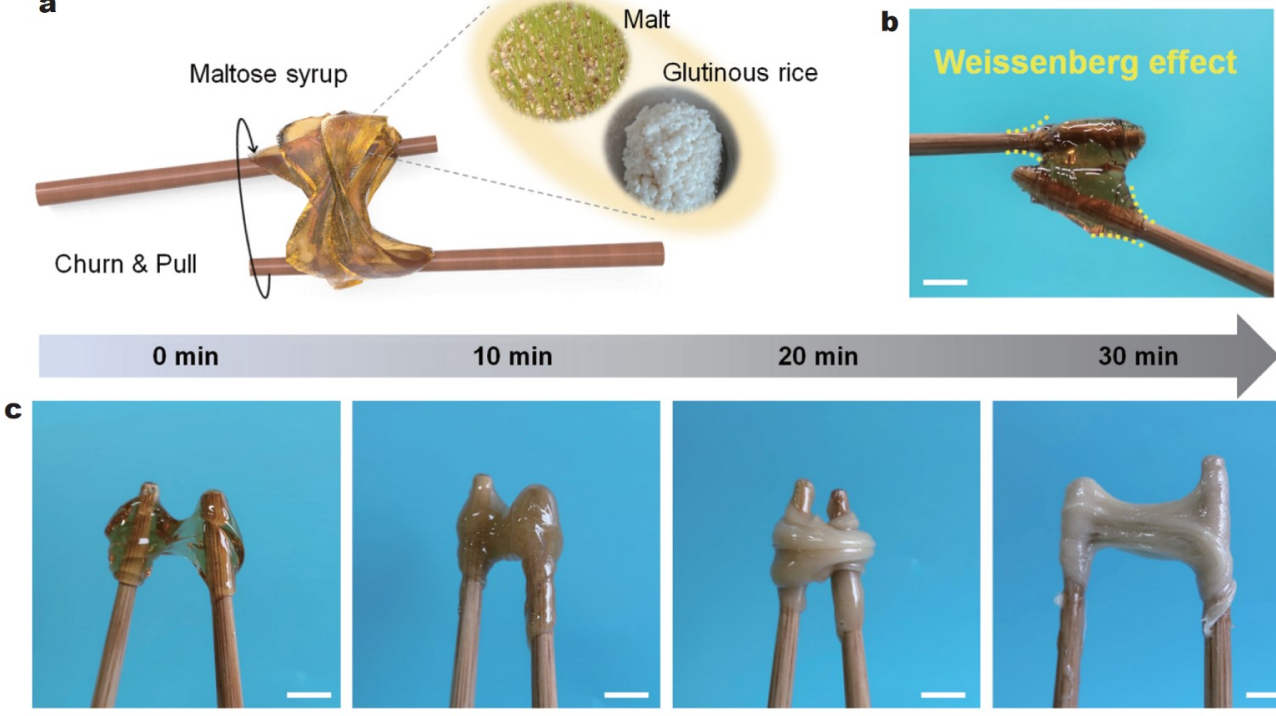

$10 \mathrm{~min}$

$20 \mathrm{~min}$

$30 \mathrm{~min}$
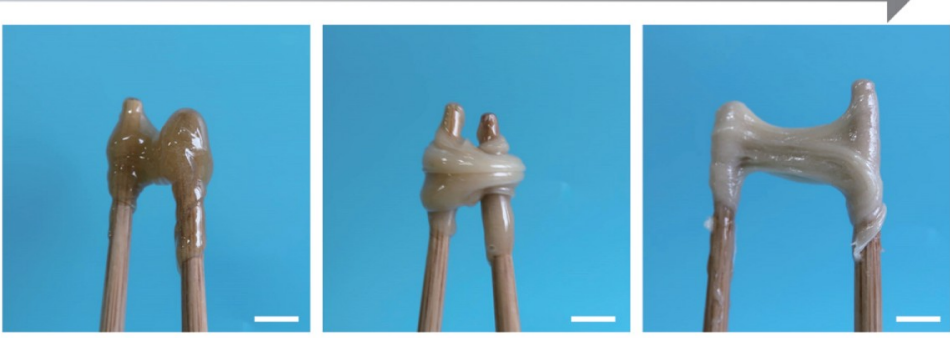

d
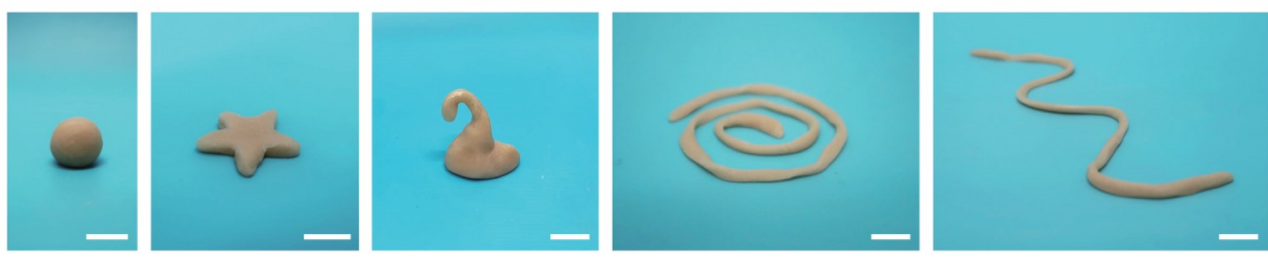

Figure 1 Introduction of the aeration and the as-made viscoelastic aerated maltose syrup. (a) Schematic illustration of the manual aeration of maltose syrup by continuous churning and pulling with two chopsticks. (b) Weissenberg effect of maltose syrup observed during aeration. (c) Photographs of changes in the appearance of the maltose syrup at different time points as the aeration time increases. (d) After churning and pulling for $30 \mathrm{~min}$, the finally obtained aerated maltose syrup can be manipulated into various shapes. Scale bars are $1 \mathrm{~cm}$. 
grees. The color of the maltose syrup gradually changed to opaque yellow and then beige, and the volume simultaneously expanded slightly. More interestingly, the finally obtained maltose syrup, termed as aerated maltose syrup, showed the increased mechanical properties together with optimized viscoelasticity, high deformability, reconfigurability and mechanical adaptation. As shown in Fig. 1d, it can be pinched with hands into various shapes, such as sphere, pentagram, swan, and spiral, like silly putty or playdough $[32,33]$. Also, when the aerated maltose syrup is sandwiched between two acrylic elastomer tapes (VHB 4905, 3M), a tight interface contact is maintained even when the material is stretched (Fig. S3).

Then, we probed into the mechanism of mechanical enhancement and microstructural evolution of maltose syrup with aeration (Fig. 2a). The mechanical improve- ment was also confirmed with rheological measurements. Fig. $2 b$ shows that at a fixed shear rate, the viscosity of maltose syrup changes with shear time, which is positively correlated and behaving shear reinforcement. Besides, dynamic frequency sweep tests at different periods were further conducted (Fig. 2c). Before and in the early stage of aeration, the loss modulus $\left(G^{\prime \prime}\right)$ is always higher than the storage modulus $\left(G^{\prime}\right)$, indicating their viscous liquid-like state. With the aid of extended continuous aeration, the final aerated maltose syrup exhibits the highest $G^{\prime}$ and $G^{\prime \prime}$, and also shows solid-like behavior in the high frequency. These results indicate that the viscoelasticity of the maltose syrup can be optimized after aeration, which is intimately coupled with the material structure.

From a microscopic perspective, the process of aeration

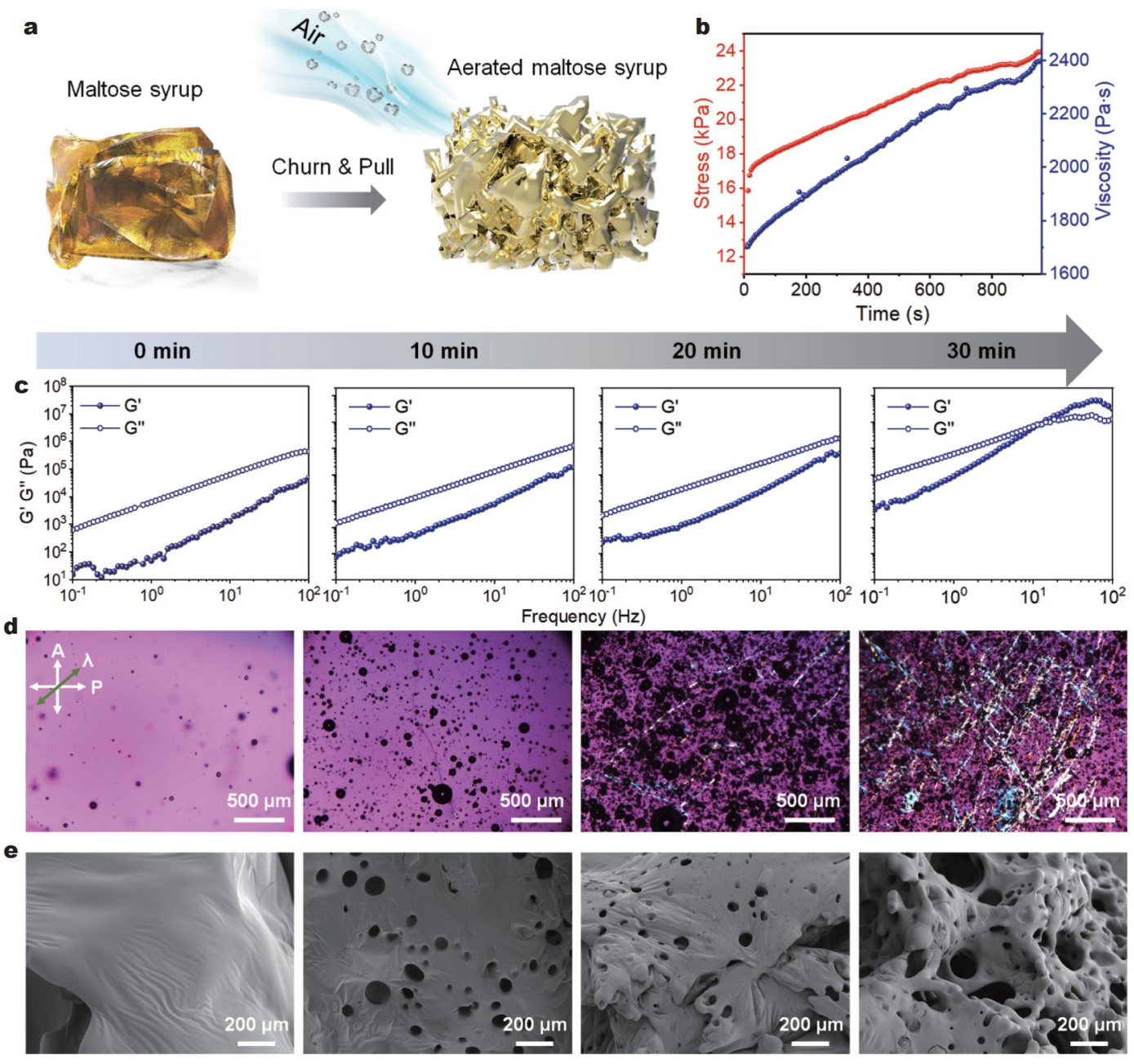

Figure 2 Characterization and analysis of structural and viscoelastic evolution during aeration. (a) Schematic illustration of the microstructural evolution of maltose syrup with aeration. (b) Viscosity measurements against time at a fixed shear rate of $1 \mathrm{~s}^{-1}$. (c) Rheological measurements of storage $\left(G^{\prime}\right)$ and loss $\left(G^{\prime \prime}\right)$ moduli. (d) POM and (e) FESEM images of maltose syrup every 10 min during the whole aeration process. 
involves two crucial steps. First, small bubbles or air cells need to be introduced, and second, those air cells should be stabilized to prevent their coalescence [28,29]. Maltose syrup is a colloidal polydisperse system, the continuous phase of which is composed of amorphous sugar and sometimes partially crystalline sugar, as vigorous agitation during pulling is also thought to contribute to crystal development [34]. Numerous small bubbles incorporated into this system serve as dispersion phase. As shown in Fig. 2d, the dark bubbles of polarized optical micrographs $(\mathrm{POM})$ are the air introduced into the maltose syrup. As the aeration level increases, the shear forces continually break down air bubbles into smaller and smaller sizes that are distributed throughout the continuous sugar phase. Also, more and more pull-induced microcrystals are observed under crossed polarizers with a $\lambda$ plate. It seems that chains in the maltose syrup can bundle together and form more or less ordered structures known as fibrous microcrystalline zones. The microcrystalline zones, acting as the junction zones of networks, are supposed to be formed by hydrogen bonds between carboxyl and/or hydroxyl groups in the saccharide backbone. The existence of non-covalent hydrogen bonds between hydroxyl groups is evidenced by the peaks around 1363 and $1020 \mathrm{~cm}^{-1}$ corresponding to the $-\mathrm{OH}$ and $\mathrm{C}-\mathrm{O}$ vibration, respectively (Fig. S4). These results are in accordance with FESEM images (Fig. 2e). The pristine maltose syrup shows bulk nature. When the degree of aeration increases, the microstructure has evolved intuitively and can be considered as solid foams with a highly interconnected, open-cell structure, which contributes to improving elasticity [35]. Furthermore, microcrystalline zones and macromolecular maltodextrins can also act at the airsugar interface to help stabilize the air bubbles by preventing newly formed bubbles from re-combining [34], also helping to optimize the viscoelastic response of aerated maltose syrup.

Overall, the aeration-induced structural evolution to a foam-like architecture comprising finely dispersed air bubbles within a sugar continuous phase enables optimized viscoelasticity.

\section{Long-term stability and transient behavior of the aerated maltose syrup}

When it comes to practicality and long-term usability, the biggest problem in food storage is spoilage, which is often caused by dehydration and the multiplication of microorganisms [25]. Meanwhile, compared to other hydrogelbased devices that are prone to dehydration under ambient conditions within several hours or days, it is still challenging for current hydrogel sensors to realize water retention capacity and long-term durability [36]. Fortunately, benefitting from the low hygroscopicity and high osmotic pressure of maltose syrup, it can keep stable with specific water content (usually $6 \%-12 \%$ ), and inhibit the growth and reproduction of microorganisms [34]. The long-term stability of the aerated maltose syrup was certificated by the fact that there was no visible deterioration after prolonged storage in normal condition $\left(22^{\circ} \mathrm{C}, 40 \% \mathrm{RH}\right)$ for 10 days (Fig. 3a). The weight of the aerated maltose syrup was also measured at different time intervals, showing minor drift $(<2 \%$, Fig. $3 b)$. The value changes of $G^{\prime}$ and $G^{\prime \prime}$ tested by dynamic frequency sweep measurements display a slight rise (Fig. S5), which confirms the viscoelasticity retention. In addition, it is well known that maltose syrup is a common food additive with excellent water solubility. The time-sequential dissolution process of the aerated maltose syrup in hot water (ca. $60^{\circ} \mathrm{C}$, left) and cold water $\left(\mathrm{ca} .15^{\circ} \mathrm{C}\right.$, right) is shown in Fig. 3c. The aerated maltose syrup exhibits an ultra-short transient behavior, dissolving and disappearing in hot water within $2.5 \mathrm{~min}$, faster than in cold water, which takes about $18 \mathrm{~min}$. Therefore, combining long-term stability and short transient behavior, as well as low preparation cost, high biodegradability and reconfigurability, the aerated maltose syrup is a promising candidate for fabricating next-generation "green" smart devices in comparison with other active materials used for electronics and iontronics such as gelatin biogel [18] and polyvinyl alcohol (PVA) composite [37] (Table S1).

\section{Aerated maltose syrup-based skin-like iontronics}

The appropriate viscoelastic properties of the aerated maltose syrup, neither too viscous to recover nor too elastic to shape, are indispensable for the application of the skin-like iontronics. Generally, "electronics" function by building reliable conductance of electronic circuits and elements, in which free electrons act as mobile charge carriers [2]. While "iontronics" function by sophisticated control of ions as signal carriers that bridges solid-state electronics and biological systems $[38,39]$. As a proof-ofconcept demonstration, we fabricated a capacitive skinlike device using a parallel-plate capacitance configuration for strain sensors, where the aerated maltose syrup and VHB tape served as the conductive layer and the dielectric layer, respectively (Fig. 4a). The capacitive signals were sensitive to strain stimuli based on geometry changes and behaved no inherent temperature sensitivity [40]. Notably, to endow the aerated maltose syrup with ionic conductivity, we introduced ca. $2 \mathrm{wt} \% \mathrm{NaCl}$ by 
a

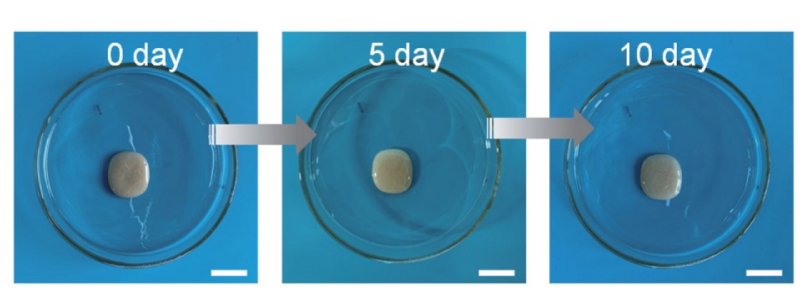

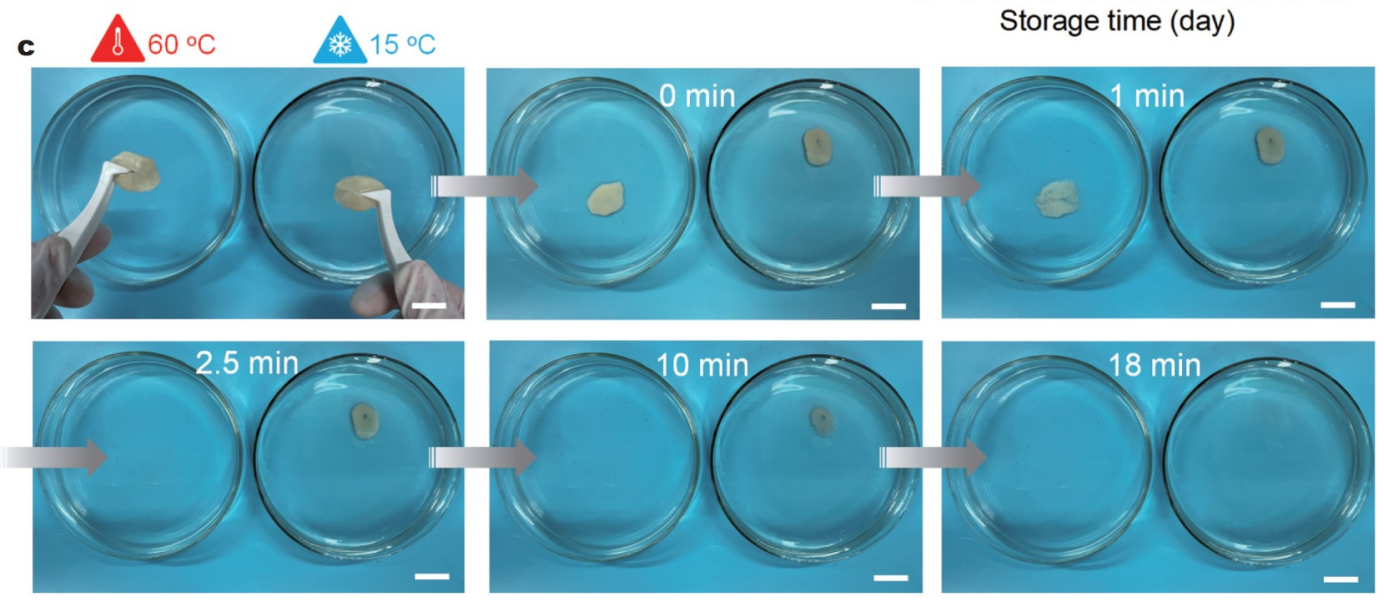

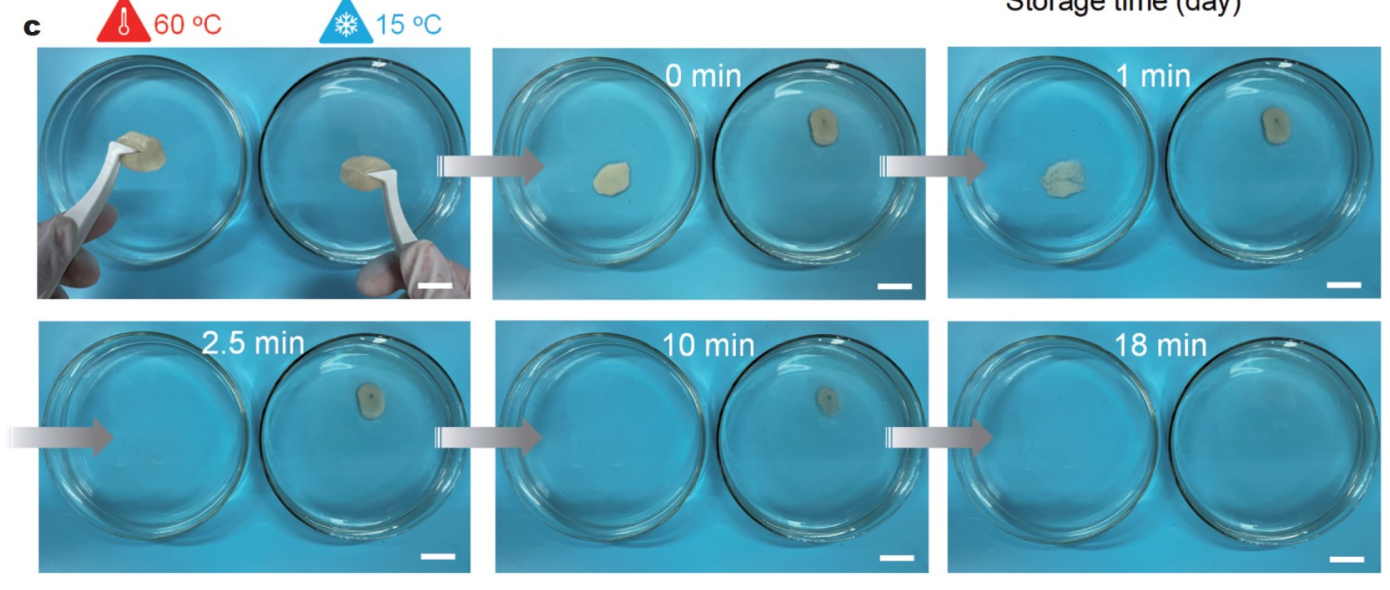

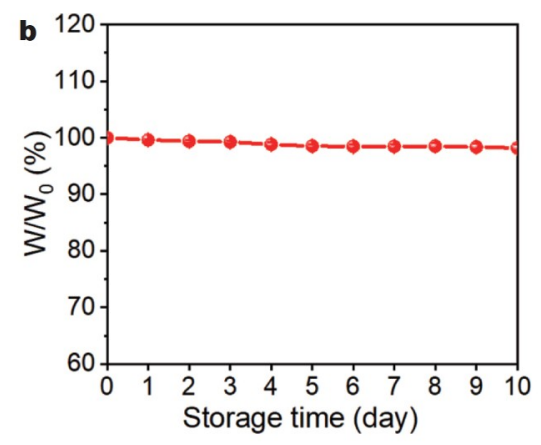

Storage time (day)

Figure 3 Long-term stability and transient behavior of the aerated maltose syrup. (a) Photographs of aerated maltose syrup in the ambient environment $\left(22^{\circ} \mathrm{C}, 40 \% \mathrm{RH}\right)$ for a long time. Scale bars are $1 \mathrm{~cm}$. (b) Weight change of the aerated maltose syrup as a function of time. $W_{0}$ is the initial weight. $W$ is the weight at different time. (c) Optical images showing the time-sequential dissolution of the aerated maltose syrup in the hot water $\left(\mathrm{ca} .60^{\circ} \mathrm{C}\right.$, left) and cold water $\left(\mathrm{ca} .15^{\circ} \mathrm{C}\right.$, right), respectively. Scale bars are $2 \mathrm{~cm}$.

kneading, similar to those that impart free ions to the hydrogel-based ionic sensors [41,42]. In Fig. 4b, under the $100 \%$ tensile strain, it shows an almost linear and reversible change in capacitive signals with a strain sensitivity of $100 \%$ during the stretching and recovery, even at different deformation frequencies (Fig. 4c). Moreover, when subjected to different tensile strains (Fig. 4d, e), an almost linear relationship between the relative capacitance and strain changes and high strain sensitivity of $100 \%$ are also observed, matching well with a theoretical prediction of $C=C_{0} \lambda$ ( $C_{0}$ and $\lambda$ refer to the initial capacitance and stretch factor, respectively) [22].

Furthermore, the incorporation of autonomous selfhealing capability into electronic devices would enhance their robustness and durability, making them advantageous in long-term use [43]. The excellent self-healing ability of the aerated maltose syrup is well demonstrated when integrated into a capacitive sensor. When two fractured parts of syrup contact, they could autonomously repair (Fig. 4f-h). The resulting healed aerated maltose syrup-based skin-like iontronics displays a higher capacitance owing to the area increase (Fig. 4i), which can also be applied as a finger motion monitor with stable and reproducible real-time capacitive electrical signals (Fig. $4 \mathrm{j}$ and Movie S2).

The credible strain sensing ability by generating a sequence of on-off signals from finger bending makes the aerated maltose syrup-based skin-like iontronics a promising platform for intelligent information encryption and transmission applications. International Morse code is a character encoding language used in telecommunication that encodes text information as standardized sequences of short and long signals called "dots" and "dashes" in various forms, such as on-off tones, lights, clicks, or electrical signals (Fig. 5a) [44-46]. By using these concepts (Fig. 5b, c), we fixed the sensor between two layers of VHB tape, and then attached it to a finger. Finger bending led to an increase in capacitance, and keeping straight made the capacitance recover to the initial state. The sensitivity and stability of our sensor guarantee the clear distinction of different stages in the information transmission, including finger bending, holding and recovery. Thus, the hold time for rhythmical finger bending could be accurately recorded, and a cipher 

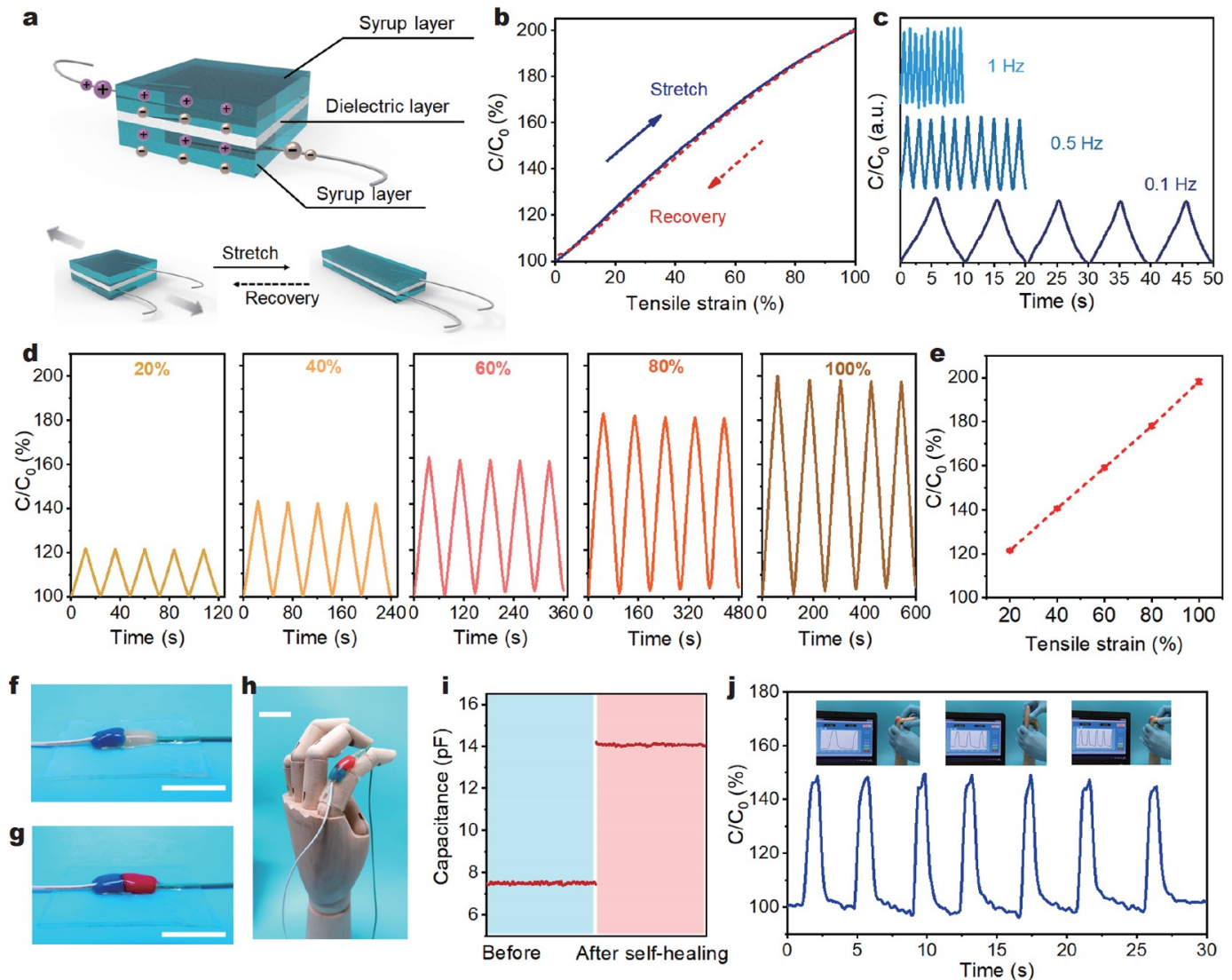

Figure 4 Properties of the aerated maltose syrup-based skin-like iontronics. (a) Schematic design of the aerated maltose syrup-based skin-like iontronics. (b) The reversible capacitance-tensile strain curves of the aerated maltose syrup-based skin-like iontronics. (c) Time-dependent relative capacitive changes of the iontronics with different frequencies under the applied tensile strain of $100 \%$. (d) The time-dependent capacitive changes at different strains. (e) Average capacitance changes at various strain changes. (f) A photo of a fractured and (g) the autonomously healed aerated maltose syrup-based skin-like iontronics (food dyes were introduced to produce the colors shown). (h) A photo of the self-healed aerated maltose syrup-based skin-like iontronics attached to a bent finger with the help of VHB tape. (i) The capacitance of the aerated maltose syrup-based skin-like iontronics before and after healing, corresponding to (f) and (g), respectively. (j) Real-time capacitive signals recorded when the finger was bent cyclically with the self-healed aerated maltose syrup-based skin-like iontronics. Scale bars are $2 \mathrm{~cm}$. The inset photos are from Movie S2.

table for encryption and decryption of the English letters of Morse code is formed (Fig. 5d). Based on the above design and unified regulations (Fig. S6), our device can effectively encode various Morse code information of alphabets without evident hysteresis, such as the examples of "SKIN" and "SYRUP" (Fig. 5e, f and Movies S3, S4). To further verify the ability of our device to transmit more complex information, "sweet" encoded confessions such as "I LOVE YOU", "YOU COMPLETE ME" and "WOULD YOU MARRY ME" were transmitted successfully (Fig. 5g and Fig. S7), by recording electrical signals of dashes and dots in different combinations.

Overall, the credible performance, good flexibility, and ease of fabrication of our aerated maltose syrup-based skin-like iontronics not only provide a valid way to recognize the information storage and decoding, but also promise its wide potential applications in wearable electronics.

\section{CONCLUSIONS}

In summary, to address the increasing concern about environmental issues and enrich the material choices for burgeoning electronics, we report a type of "green" skinlike iontronics based on aerated maltose syrup, which is mechanically flexible and conformal, viscoelastic, moldable and self-healing, yet with transient behavior. Moreover, we analyze the structural and viscoelastic evolution during aeration to understand its structure-property relationships. For future large-scale applications, machine aeration could provide a more efficient means. Finally, a new Morse code transmission system using the "green" skin-like iontronics is developed and demonstrated for 

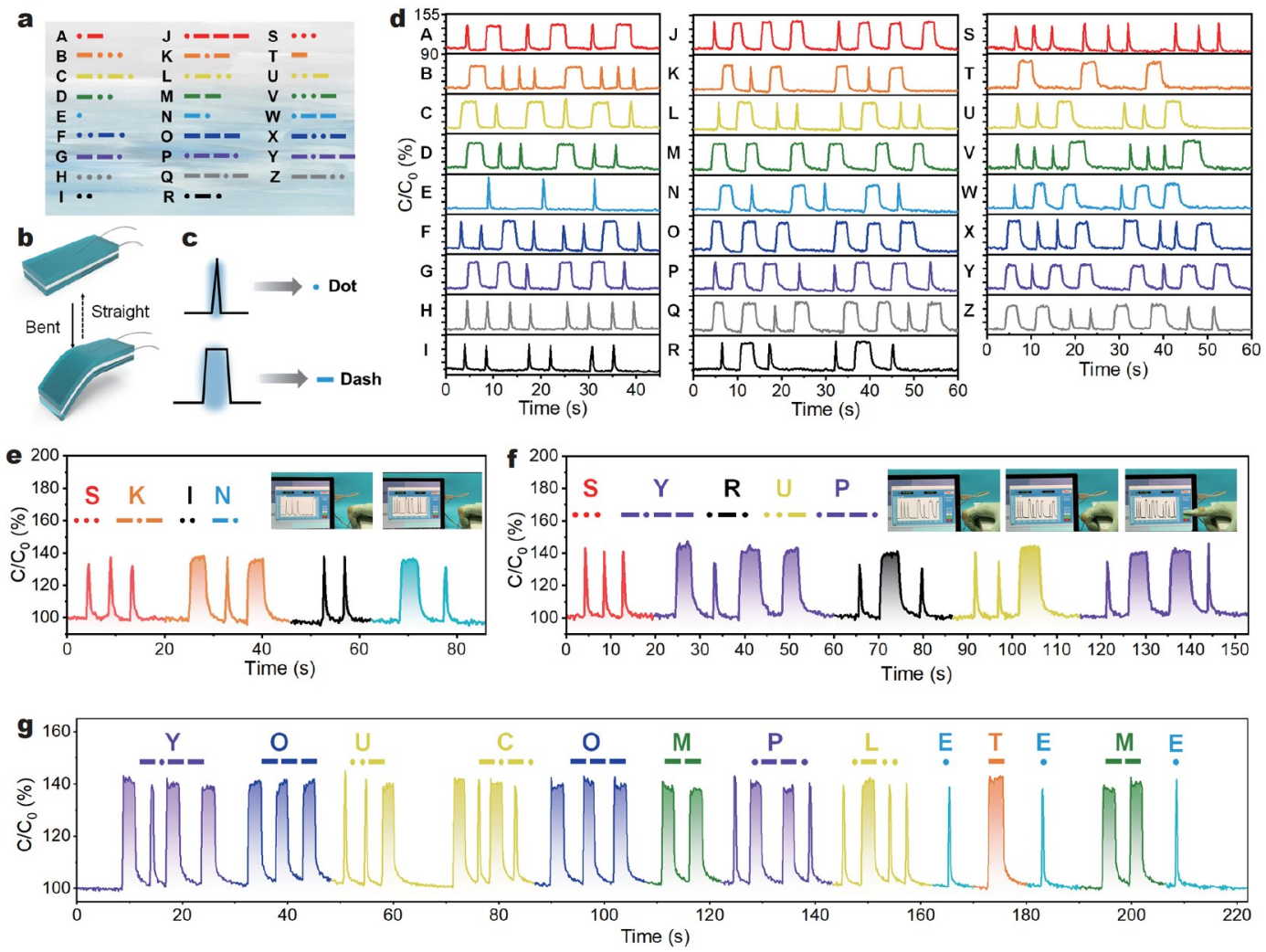

Figure 5 Proof-of-concept demonstration of the aerated maltose syrup-based iontronics as a kind of "sweet" Morse code encoder. (a) International Morse code table of English letters. (b) Schematic diagram of the aerated maltose syrup-based skin-like iontronics used as a Morse code encoder. (c) Definition of dot and dash as capacitive signals of instant and lasting bending of a finger, respectively. (d) A cipher table that encodes English letters by capacitive signals. Representative encrypted examples of (e) "SKIN" and (f) "SYRUP" using Morse code. The inset photos are from Movies S3 and S4, respectively. (g) "Sweet" Morse code example of "YOU COMPLETE ME".

real-time messaging. We believe that food-based materials would extend the material choices as new biodegradable and environmentally friendly materials for electronics or iontronics. Continued exploration of additional functional materials from food will further enrich and shed light on this field for broader sensing and actuation capability.

\section{Received 18 March 2021; accepted 7 May 2021; published online 15 July 2021}

1 Huang S, Liu Y, Zhao Y, et al. Flexible electronics: Stretchable electrodes and their future. Adv Funct Mater, 2019, 29: 1805924

2 Yuk H, Lu B, Zhao X. Hydrogel bioelectronics. Chem Soc Rev, 2019, 48: 1642-1667

3 Jian M, Wang C, Wang Q, et al. Advanced carbon materials for flexible and wearable sensors. Sci China Mater, 2017, 60: 10261062

4 Forrest SR. The path to ubiquitous and low-cost organic electronic appliances on plastic. Nature, 2004, 428: 911-918

5 Fu KK, Wang Z, Dai J, et al. Transient electronics: Materials and devices. Chem Mater, 2016, 28: 3527-3539

6 Irimia-Vladu M. "Green" electronics: Biodegradable and biocompatible materials and devices for sustainable future. Chem Soc
Rev, 2014, 43: 588-610

7 Wang L, Chen D, Jiang K, et al. New insights and perspectives into biological materials for flexible electronics. Chem Soc Rev, 2017, 46: 6764-6815

8 Wang L, Wang K, Lou Z, et al. Plant-based modular building blocks for "green" electronic skins. Adv Funct Mater, 2018, 28: 1804510

9 Marr PC, Marr AC. Ionic liquid gel materials: Applications in green and sustainable chemistry. Green Chem, 2016, 18: 105-128

10 Li W, Liu Q, Zhang Y, et al. Biodegradable materials and green processing for green electronics. Adv Mater, 2020, 32: 2001591

11 Teng L, Ye S, Handschuh-Wang S, et al. Liquid metal-based transient circuits for flexible and recyclable electronics. Adv Funct Mater, 2019, 29: 1808739

12 Son D, Lee J, Lee DJ, et al. Bioresorbable electronic stent integrated with therapeutic nanoparticles for endovascular diseases. ACS Nano, 2015, 9: 5937-5946

13 Yin R, Yang S, Li Q, et al. Flexible conductive Ag nanowire/cellulose nanofibril hybrid nanopaper for strain and temperature sensing applications. Sci Bull, 2020, 65: 899-908

14 Wang $\mathrm{C}$, Hou X, Cui M, et al. An ultra-sensitive and wide measuring range pressure sensor with paper-based CNT film/interdigitated structure. Sci China Mater, 2020, 63: 403-412

15 Zhu B, Wang H, Leow WR, et al. Silk fibroin for flexible electronic devices. Adv Mater, 2016, 28: 4250-4265 
16 Wang Q, Ling S, Liang X, et al. Self-healable multifunctional electronic tattoos based on silk and graphene. Adv Funct Mater, 2019, 29: 1808695

17 Wang C, Xia K, Zhang Y, et al. Silk-based advanced materials for soft electronics. Acc Chem Res, 2019, 52: 2916-2927

18 Baumgartner M, Hartmann F, Drack M, et al. Resilient yet entirely degradable gelatin-based biogels for soft robots and electronics. Nat Mater, 2020, 19: 1102-1109

$19 \mathrm{Xu} \mathrm{W}$, Yang H, Zeng W, et al. Food-based edible and nutritive electronics. Adv Mater Technol, 2017, 2: 1700181

20 Chang Q, He Y, Liu Y, et al. Protein gel phase transition: Toward superiorly transparent and hysteresis-free wearable electronics. Adv Funct Mater, 2020, 30: 1910080

21 Darabi MA, Khosrozadeh A, Wang Q, et al. Gum sensor: A stretchable, wearable, and foldable sensor based on carbon nanotube/chewing gum membrane. ACS Appl Mater Interfaces, 2015, 7: 26195-26205

22 Lei $\mathrm{Z}$, Huang J, Wu P. Traditional dough in the era of internet of things: Edible, renewable, and reconfigurable skin-like iontronics. Adv Funct Mater, 2020, 30: 1908018

23 Cao Y, Mezzenga R. Design principles of food gels. Nat Food, 2020, 1: $106-118$

24 Wang X, Xu W, Chatterjee $\mathrm{P}$, et al. Food-materials-based edible supercapacitors. Adv Mater Technol, 2016, 1: 1600059

25 Powrie WD. Chemical effects during storage of frozen foods. J Chem Educ, 1984, 61: 340

26 Lin Q, Xiao H, Liu GQ, et al. Production of maltose syrup by enzymatic conversion of rice starch. Food Bioprocess Technol, 2013, 6: 242-248

27 Saha BC, Zeikus JG. Improved method for preparing high maltose conversion syrups. Biotechnol Bioeng, 1989, 34: 299-303

28 Zúñiga R, Aguilera J. Aerated food gels: Fabrication and potential applications. Trends Food Sci Tech, 2008, 19: 176-187

29 Campbell G. Creation and characterisation of aerated food products. Trends Food Sci Tech, 1999, 10: 283-296

30 Campbell GM. Chapter 1-A history of aerated foods. In: Campbell GM, Scanlon MG, Pyle DL (eds.). Bubbles in Food 2. St. Paul: Woodhead Publishing and AACC International Press, 2008. 1-21

31 Weissenberg K. A continuum theory of rhelogical phenomena. Nature, 1947, 159: 310-311

32 Boland CS, Khan U, Ryan G, et al. Sensitive electromechanical sensors using viscoelastic graphene-polymer nanocomposites. Science, 2016, 354: 1257-1260

33 Suh D, Faseela KP, Kim W, et al. Electron tunneling of hierarchically structured silver nanosatellite particles for highly conductive healable nanocomposites. Nat Commun, 2020, 11: 2252

34 Hartel RW, von Elbe JH, Hofberger R. Aerated confections. In: Hartel RW, von Elbe JH, Hofberger R (eds.). Confectionery Science and Technology. Cham: Springer, 2018. 301-327

35 Wang J, Kliks MM, Jun S, et al. Rapid analysis of glucose, fructose, sucrose, and maltose in honeys from different geographic regions using Fourier transform infrared spectroscopy and multivariate analysis. J Food Sci, 2010, 75: C208-C214

36 Liu X, Liu J, Lin S, et al. Hydrogel machines. Mater Today, 2020, 36: $102-124$

37 Fu X, Li J, Tang C, et al. Hydrogel cryo-microtomy continuously making soft electronic devices. Adv Funct Mater, 2020, 31: 2008355

38 Chun H, Chung TD. Iontronics. Annu Rev Anal Chem, 2015, 8: 441-462
39 Yang C, Suo Z. Hydrogel ionotronics. Nat Rev Mater, 2018, 3: 125142

40 Chortos A, Bao Z. Skin-inspired electronic devices. Mater Today, 2014, 17: 321-331

41 Bai Y, Chen B, Xiang F, et al. Transparent hydrogel with enhanced water retention capacity by introducing highly hydratable salt. Appl Phys Lett, 2014, 105: 151903

42 Bai J, Wang R, Ju M, et al. Facile preparation and high performance of wearable strain sensors based on ionically cross-linked composite hydrogels. Sci China Mater, 2021, 64: 942-952

43 Someya T, Bao Z, Malliaras GG. The rise of plastic bioelectronics. Nature, 2016, 540: 379-385

44 Chandrasekhar A, Vivekananthan V, Khandelwal G, et al. A fully packed water-proof, humidity resistant triboelectric nanogenerator for transmitting Morse code. Nano Energy, 2019, 60: 850-856

45 Liu S, Cheng Y, Li Y, et al. Manipulating solid-state intramolecular motion toward controlled fluorescence patterns. ACS Nano, 2020, 14: 2090-2098

46 Qin R, Liu Y, Tao F, et al. Protein-bound freestanding 2D metal film for stealth information transmission. Adv Mater, 2018, 1803377

Acknowledgements This work was supported by the National Natural Science Foundation of China (51733003).

Author contributions Huang $\mathrm{J}$ and $\mathrm{Wu} \mathrm{P}$ designed the experiments. Huang J performed the experiments. Huang $\mathrm{J}$ and $\mathrm{Wu} \mathrm{P}$ analyzed the data and wrote the paper.

Conflict of interest The authors declare that they have no conflict of interest.

Supplementary information online version of the paper.

Supporting data are available in the

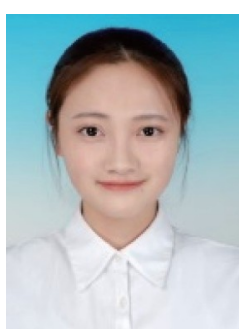

Jiahui Huang received her BSc degree in textile chemistry and dyeing and finishing engineering at Donghua University in 2018 and currently is a $\mathrm{PhD}$ candidate at Fudan University. Her research mainly focuses on flexible hydrogel electronics and $3 \mathrm{D}$ printing multifunctional materials.

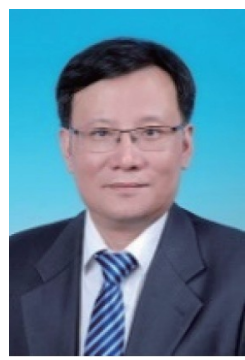

Peiyi $\mathbf{W u}$ is a professor at the College of Chemistry, Chemical Engineering and Biotechnology, Donghua University (China) and a fellow of the Royal Society of Chemistry. He received his $\mathrm{PhD}$ degree from the University of Essen (Germany) in 1998. His research interests are focused on the 2D-IR spectroscopy, flexible hydrogel electronics and iontronics, and the synthesis and application of 2D materials. 


\section{基于用筷子改造的传统甜食“搅摚糖”(麦芽糖浆) 构筑的离子皮肤器件}

黄佳惠 ${ }^{1}$, 武培怡 ${ }^{1,2 *}$

摘要 科技的发展对生活的各个领域都产生了深远的影响, 使得数 字化领域和生物领域的结合越来越紧密, 但同时也可能伴随着电 子设备和石化产品的滥用所带来的环境危机. 具有高生物相容 性、生物降解性、低成本和顺应性的下一代“绿色”电子有望缓解 这些不利影响, 但往往受限于材料和策略的选择. 本工作利用传统 甜食“摚摚糖”(麦芽糖浆), 使用筷子对其进行结构改造, 即所谓的 充气，充气后的麦芽糖浆具有独特的黏弹性、力学适应性、可重 构性和自修复能力, 但又兼具瞬态行为, 可以替代当前电子器件中 不可回收的导电元件. 此外, 本文还分析了充气过程中结构和潻弹 性的演变. 作为概念验证, 本文中制备的电容式离子皮肤具有可靠 的应变传感能力, 可应用于新型的信息加密和传输. 这项工作将传 统食物与前沿的科学研究相结合, 极大地拓展了材料的选择性, 有 望为未来材料的可持续发展提供新的思路. 